Gynäkologische Endokrinologie 2006 · 4:52-64 DOI 10.1007/s10304-006-0139-8

Online publiziert: 4. Februar 2006

๑) Springer Medizin Verlag 2006

\section{Redaktion}

W. Braendle, Hamburg

T. Gudermann, Marburg

O. Ortmann, Regensburg

M. Birkhäuser • Universitäts-Frauenklinik, Inselspital, Bern

\title{
Klinische Bedeutung von gestagenen Partialwirkungen
}

\section{Wirkungsmechanismen}

moleküle definiert worden, welche die Schwangerschaft aufrechterhalten können. Es hat sich allerdings erwiesen, dass beim Menschen nur das natürliche Progesteron diese Fähigkeit besitzt. Die hauptsächliche Rolle der Gestagene, insbesondere im Rahmen der Hormonersatztherapie, ist daher heute die Hemmung der östrogeninduzierten Endometriumproliferation und die sekretorische Umwandlung des unter Östrogeneinfluss proliferierten Endometriums.
Die Wirkung von Progesteron und der synthetischen Gestagene wird durch eine genomische Interaktion mit dem Progesteronrezeptor einerseits und einer raschen nichtgenomischen Interaktion mit membranären Bindungsstellen andererseits vermittelt. Der Progesteronrezeptor existiert in 2 Isoformen, PRA und PRB.

Progesteron (P) und synthetische Gestagene interagieren aber nicht allein mit dem Progesteronrezeptor (PR): Entsprechend dem ursprünglichen Molekül, von dem sich ein synthetisches Gestagen ableitet (Testosteron oder Progesteron), und gemäß ihrer spezifischen chemischen und räumlichen Struktur binden bestimmte Gestagene auch an andere Steroidrezeptoren, z. B. den Androgenrezeptoren (AR), wo sie eine androgene oder eine antiandrogene Partialwirkung zeigen, oder an den Mineralkortikoidrezeptoren (MR), wo sie zu einer kompetitiven Hemmung des Aldosterons führen. Einige Gestagene besitzen auch eine Affinität zum Glukokortikosteroidrezeptor (GR) und können somit eine gewisse kortisonähnliche Wirkung auslösen.

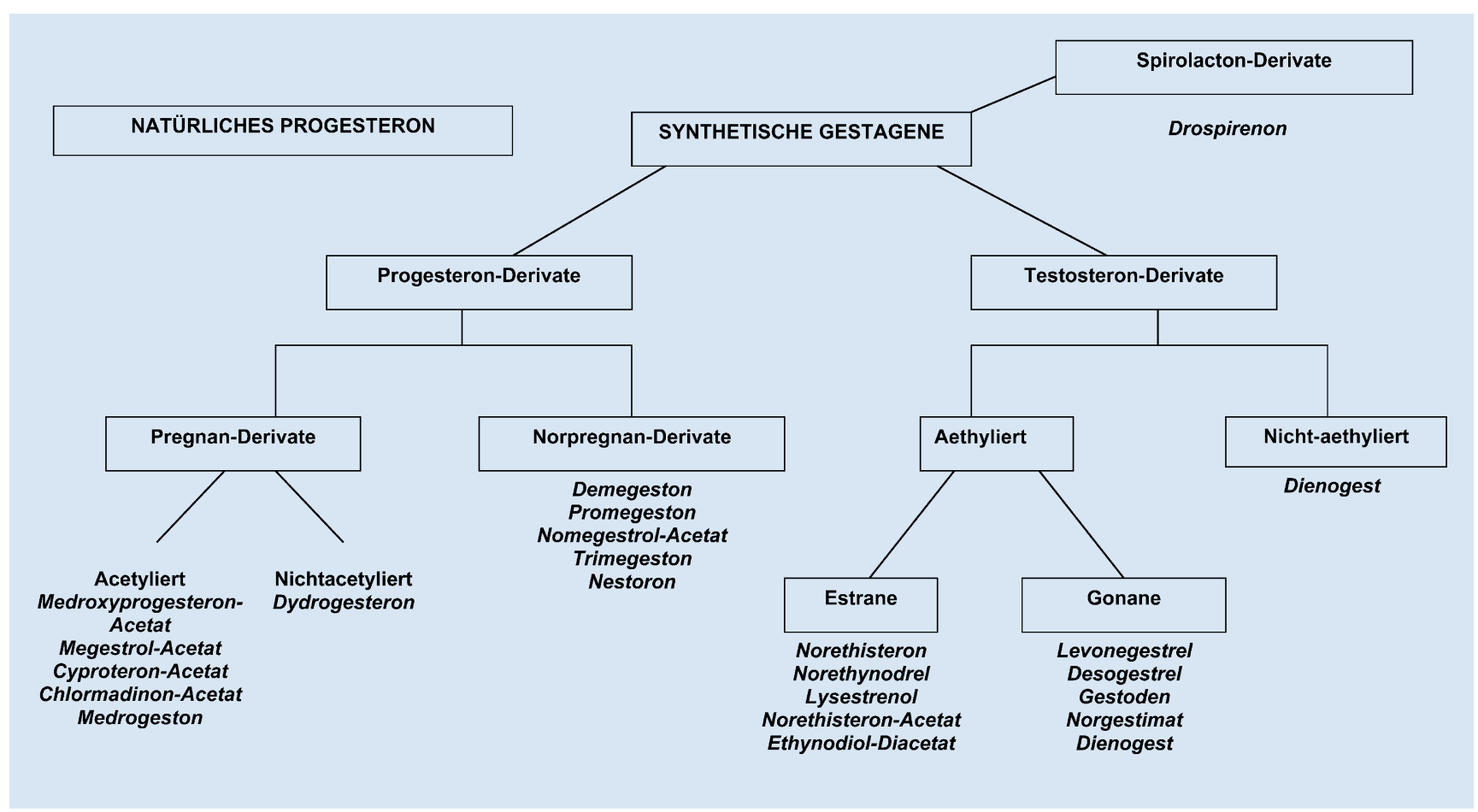

Abb. $1 \Delta$ Klassifizierung der Gestagene 
Die biologische Wirkung der Gestagene ist in den meisten Geweben von einer Östrogenpräsenz abhängig, da Östrogene für die Induktion von Progesteronrezeptoren entscheidend sind. Umgekehrt wird die Expression von Östrogenrezeptoren durch Gestagene herunterreguliert. Im Endometrium können Gestagene zudem die eigenen Progesteronrezeptoren hemmen. Hingegen hemmen in der menschlichen Brust Gestagene - anders als im Endometrium - die östrogeninduzierte Proliferation des Brustepithels nicht, sondern fördern diese zusätzlich, obwohl auch in der Brust die Östrogen- und Progesteronrezeptoren downreguliert werden [11].

\section{Einteilung der Gestagene}

Die in der klinischen Praxis verwendeten synthetischen Gestagene (• Abb. 1) werden von natürlichen Sexualsteroiden (- Abb. 2) abgeleitet, entweder von Progesteron/OH-Progesteron (17- $\alpha$-Hydroxy-Progesteron-Derivate und 19-Nor-Progesteron-Derivate) oder von Testosteron (19-Nortestosteronderivate) [23].

\section{7-a-OH-Progesteron-Derivate}

Die 17- $\alpha$-OH-Progesteron-Derivate werden klinisch v. a. zur hormonalen Substitution und - entsprechend ihrer Partialwirkung - als Antiandrogene eingesetzt. Zur Gruppe der 17- $\alpha$-OH-ProgesteronDerivate gehören einerseits die Pregnane (Medroxy-Progesteron-Acetat=MPA, Megestrol-Acetat, Medrogeston und Dydrogesteron, sowie die Antiandrogene Cyproteron-Acetat $=$ CPA und ChlormadinonAcetat=CMA), andererseits die Norpregnane (Demegeston, Promegeston, Trimegeston und Nestoron). NomegestrolAcetat ist kein eigentliches Pregnan, wird von seinem Wirkungsprofil aber meist mit dieser Gruppe genannt. Die Pregnane werden weltweit v. a. entweder zur Hormonersatztherapie (HRT) nach der Menopause (z. B. MedroxyprogesteronAcetat und Dydrogesteron) oder therapeutisch bei Frauen mit Androgenisierungserscheinungen als Antiandrogene verwendet. Das potenteste Antiandrogen ist Cyproteron-Acetat. Die Norpregnane werden bisher fast ausschließlich in Frankreich eingesetzt, obwohl es sich

Gynäkologische Endokrinologie 2006 · 4:52-64

DOI 10.1007/s10304-006-0139-8

○) Springer Medizin Verlag 2006

\section{Birkhäuser \\ Klinische Bedeutung von gestagenen Partialwirkungen}

\section{Zusammenfassung}

Die Partialwirkungen der zur Kontrazeption und zur Hormonersatztherapie eingesetzten Gestagene sind nicht dieselben. Sie unterscheiden sich hinsichtlich ihrer Struktur, ihrer Metabolisierung, ihrer Bioverfügbarkeit und ihrer Partialwirkungen. Deshalb ist es unsinnig, die verschiedenen Gestagene mit ihren unterschiedlichen Wirkungsprofilen in eine einheitliche Klasse einzuteilen. Entsprechend ihrer Partialwirkungen werden die Gestagene heute gezielt zur Kontrazeption, zur postmenopausalen Hormontherapie oder als Antian- drogen eingesetzt. Neuere von ihrem Wirkungsprofil her günstige Gestagene sind die Norpregnane, Dienogest und Drospirenon. Diese Übersicht fasst die Einteilung der Gestagene, deren wichtigste Merkmale und Partialwirkungen und deren mögliche Folgen für die Klinik zusammen.

\section{Schlüsselwörter}

Antiandrogen · Kontrazeption ·

Hormonersatztherapie · Partialwirkungen . Gestagene

\section{Clinical significance of partial gestagen effects}

\section{Abstract}

The partial effects of progestagens used for contraception and for hormone replacement therapy are not the same. They differ in their structure, metabolization, bioavailability, and partial effects. Thus, it is absurd to classify the various gestagens with their different activity profiles into one uniform category. Nowadays, progestagens are specifically employed for contraception, postmenopausal hormone therapy, or as antiandrogens depending on their partial effects. Newer progestagens that are more beneficial based on their activity profile include norpregnane, dienogest, and drospirenone. This overview summarizes the classification of progestagens, their most important characteristics, and their partial effects as well as possible consequences for clinical practice.

\section{Keywords}

Antiandrogens · Contraception · Hormone replacement therapy · Partial effects . Gestagens 

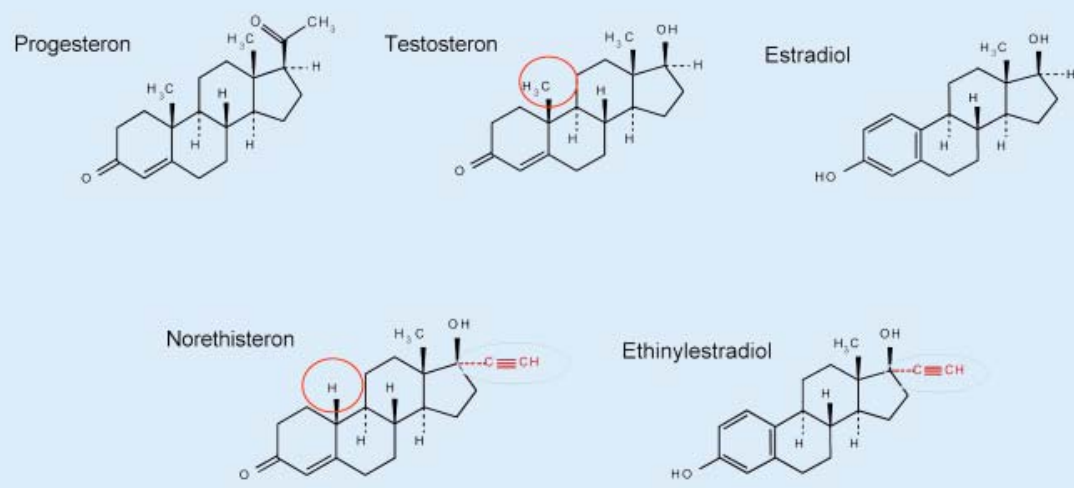

Abb. $2 \Delta$ Körpereigene und synthetische Sexualhormone

um von ihrem Profil her klinisch günstige Gestagene handelt [50].

\section{9-Nortestosteronderivate}

Die 19-Nortestosteronderivate verlieren, obwohl vom Androgen 19-Nortestosteron abgeleitet, durch das Einführen einer Ethinylgruppe an Position C-17- $\alpha$ ihre androgene Wirkung zugunsten einer gestagenen Aktivität. Sie werden in eine 1., 2. und 3. Generation eingeteilt. Zur 1. Generation gehört Norethinodrel, das erste synthetische Gestagen [23]. Die 2. Generation gliedert sich wiederum in 2 Gruppen: die Estrane, welche Norethisteron (=Norethindron=NET) und seine Metaboliten einschließen, und die Gonane, welche aus Levonorgestrel (LNG) und seinen Derivaten bestehen. Norethinodrel, Lynestrenol und Ethynoldiolacetat sind Prodrugs von Norethisteron und werden im Körper in NET umgewandelt und dadurch aktiviert. Norethisteron-Acetat ist eines der v. a. in Europa in der postmenopausalen Hormonsubstitution am häufigsten verwendeten Gestagene. Die 19Nortestosteronderivate der 3. Generation sind vom Norgestrel abgeleitet und werden überwiegend zur hormonalen Kontrazeption verwendet. Zur 3. Generation gehören Desogestrel (DSG) mit seinem aktiven Metaboliten 3-Keto-Desogestrel oder Etonegestrel, Gestoden (GES), Dienogest und Norgestimat, dessen aktiver Metabolit, Norelgestromin, an Position 17 deazetyliert ist. Diese vom Testosteron abgeleiteten Gestagene werden in den meisten heute verfügbaren oralen Kontrazeptiva eingesetzt und besitzen z. T. noch eine androgene Partialwirkung.

\section{Pharmakologische Unterschiede}

Die pharmakokinetischen Eigenschaften und die Bindung von Gestagenen an die Serumproteine bilden einen weiteren wichtigen Aspekt bei der Evaluierung von Gestagenen vor ihrer klinischen Verwendung. Üblicherweise wird die Bioverfügbarkeit dadurch ermittelt, dass das zu testende Molekül in einer radioaktiv markierten Form verabreicht und die Ausscheidung in Urin und Fäzes gemessen wird. Nach dieser Methode sind Gestoden, Desogestrel und Cyproteron-Acetat die Gestagene mit der höchsten oralen Bioverfügbarkeit [13, 29]. Auch die beiden neueren Gestagene Dienogest und Promegeston besitzen eine hohe orale Bioverfügbarkeit $[18,42,45]$. Demgegenüber besitzt Nestoron eine sehr niedrige orale Bioverfügbarkeit.

Die Bindung eines Moleküls an Plasmoproteine [insbesondere an das spezifische Trägereiweiß sexhormonbindendes Globulin (SHBG) und an das unspezifisch bindende Albumin] beeinflusst maßgeblich die Halbwertszeit jedes Gestagens. Zur Ermittlung der Halbwertszeit von Gestagenen wird als Referenzsubstanz Testosteron eingesetzt. Levonorgestrel und 3-KetoDesogestrel besitzen eine signifikant niedrigere Affinität zu SHBG als Testosteron [33] und somit eine größere ungebundene Fraktion. Obwohl von den 2 synthetischen Gestagenen Norethisteron und Levenorgestrel beide an SHBG binden, ist ihre Halbwertszeit unterschiedlich. Während die terminale Halbwertszeit für Norethisteron ungefähr 7-8 $\mathrm{h}$ beträgt, liegt diejenige von Levenorgestrel bei rund $26 \mathrm{~h}$. Dienogest hat eine kurze Halbwertszeit von 6$12 \mathrm{~h}$ [29]. Eine deutlich längere Halbwerts- zeit besitzen CPA $(48 \mathrm{~h},[13,50])$ und Nomegestrol-Acetat (50 h, [14]). Bei der längeren Halbwertszeit von Progesteronderivaten gegenüber Progesteron selbst spielt auch deren Speicherung im Fettgewebe eine Rolle [29].

Progesteron, Drospirenon, Dienogest und Nestoron binden nicht an SHBG [33, $44,45,48,51,56]$, sodass deren freie biologisch aktive Fraktion größer ist als diejenige der klassischen 19-Nortestosteronderivate.

\section{Partialwirkungen der klinisch verwendeten Gestagene}

Die unterschiedlichen Bindungseigenschaften jedes einzelnen synthetischen Gestagens an die verschiedene Steroidhormonrezeptoren erklären die unterschiedlichen und spezifischen Partialwirkungen jeder Substanz (• Tabelle 1 und 2). Es ist somit falsch, bei den Gestagenen von einem Klasseneffekt zu sprechen.

- Tabelle 1 (aus [31]) gibt eine Übersicht über die hormonell wirksamen Partialwirkungen von Gestagenen im Vergleich zu Progesteron, • Tabelle 2 (nach [31]) über deren hormonale Potenz. Dabei muss berücksichtigt werden, dass diese Angaben mehrheitlich von Tierexperimenten stammen und somit nicht immer in vollem Umfang auf den Menschen übertragbar sind. Zudem hängt die beobachtete klinische Wirkung nicht nur von der Partialwirkung eines Gestagens, sondern auch von dessen Konzentration im Gewebe ab.

\section{Was sind Partialwirkungen?}

Die Bindungsaffinitäten der verschiedenen Gestagene an die klinisch relevanten Steroidrezeptoren wie Östrogenrezeptoren (ER), Androgenrezeptoren (AR), Mineralokortikoidrezeptoren (MR) und Glukokortikoidrezeptoren (GR) zeigen beträchtliche Unterschiede (• Tabelle 2). Kommt es zu einer Bindung, kann diese sowohl eine kompetitive Hemmung (antagonistische Wirkung) oder eine entsprechende Aktivierung (agonistische Wirkung) auslösen. Je nach ihrer chemischen Struktur und ihrer räumlichen Ähnlichkeit mit dem entsprechenden natürlichen Steroid (Östradiol, Testosteron, Kortisol, Aldosteron) können Gestagene als schwache Androgene oder 
Tabelle 1

Spektrum der hormonalen Aktivitäten von Gestagenen

\begin{tabular}{|c|c|c|c|c|c|c|}
\hline Gestagen & A-E & EST & AND & A-A & GLU & A-M \\
\hline Progesteron & + & - & - & $(+)$ & + & + \\
\hline Chlormadinon-Acetat & + & - & - & + & + & - \\
\hline Cyproteron-Acetat & + & - & - & + & + & - \\
\hline Medroxyprogesteron-Acetat & + & & $(+)$ & - & + & - \\
\hline Medrogeston & + & - & - & - & $?$ & - \\
\hline Dydrogesteron & + & - & - & - & $?$ & $(+)$ \\
\hline Norethisteron & + & + & + & - & - & - \\
\hline Levonorgestrel & + & - & + & - & - & - \\
\hline Gestoden & + & - & + & - & $(+)$ & + \\
\hline Etonogestrel (3-Keto-Desogestrel) & + & - & + & - & $(+)$ & - \\
\hline Norgestimat & + & - & + & - & $?$ & $?$ \\
\hline Dienogest & + & - & - & + & - & - \\
\hline Tibolon-Metaboliten & + & + & ++ & - & - & - \\
\hline Drospirenon & + & - & - & + & - & + \\
\hline Trimegeston & + & - & - & $(+)$ & - & $(+)$ \\
\hline Promegeston & + & - & - & - & + & - \\
\hline Nomegestrol-Acetat & + & - & - & + & - & - \\
\hline Nestoron & + & - & - & - & - & $?$ \\
\hline
\end{tabular}

Diese Daten stützen sich v. a. auf Tierexperimente (nach [31]). Die klinische Wirkung der Gestagene hängt von ihrer Gewebekonzentration ab. A-E antiöstrogen, EST östrogen, AND androgen, A-A antiandrogen, GLU glukokortikoide Aktivität, A-M antimineralokortikoide Aktivität. ++ stark wirksam, + wirksam, (+) schwach wirksam, - unwirksam, ? unbekannt.

Antiandrogene, als Glukokortikoide oder als Antimineralokortikoide wirken.

\section{Gestagene Potenz eines Gestagens}

Als Maßstab für die gestagene Potenz eines Gestagens können die Ovulationshemmdosis, die Transformationsdosis und die schwangerschaftserhaltende Dosis der betreffenden Substanz gelten. Die eigentliche gestagene Aktivität der verschiedenen synthetischen Gestagene wird üblicherweise mit dem McPhail-Index bei unreifen Kaninchen und durch die Bestimmung der schwangerschaftserhaltenden Wirkung bei weiblichen Ratten festgelegt. Der Ovulationshemmtest, wichtig für orale Kontrazeptiva, wird ebenfalls bei weiblichen Ratten durchgeführt und dazu eingesetzt, um die biologische antiovulatorische Potenz von synthetischen Steroidhormonen zu testen. Die Ovulationshemmdosis wird so festgelegt, dass bei Frauen mit einem gesicherten ovulatorischen Zyklus täglich eine bestimmte Gestagendosis pro Zyklustag verabreicht wird. Die niedrigste Dosis, welche die Ovulation bei allen Frauen hemmt, gilt als Ovulationshemmdosis.
Die Transformationsdosis wird bei ovariektomierten Frauen ermittelt, die zunächst über 14 Tage täglich $50 \mu \mathrm{g}$ Ethinalöstradiol (EE) allein erhalten hatten, gefolgt von 10 Tagen einer Kombination derselben EE-Dosis mit steigenden Dosierungen des zu untersuchenden Gestagens. Als Transformationsdosis gilt diejenige tägliche Gestagendosierung, welche eine volle sekretorische Transformation des proliferierten Endometriums induziert. Die hohe perorale Transformationsdosis des natürlichen Progesterons ist die Folge seiner niedrigen oralen Bioverfügbarkeit, welche wiederum die Konsequenz einer raschen Inaktivierung ist. Die relativ hohen Transformationsdosen von NETA und NET können durch die Aromatisierung einer kleinen Fraktion von NET zu EE erklärt werden. $\mathrm{EE}$ antagonisiert den gestagenen Effekt von NET im Endometrium [7, 32].

\section{Östrogene Partialwirkung von Gestagenen}

Die Untersuchung der östrogenen Partialwirkung von Gestagenen erfolgt durch die Bestimmung des Uterusgewichts von immaturen ovariektomierten weiblichen Ratten.

\section{Androgene Partialwirkung von Gestagenen}

Die gebräuchlichen Bioassays testen die androgene Partialwirkung eines Gestagens meist anhand der Gewichtszunahme der ventralen Prostata oder anderer Sexualorgane bei der unreifen männlichen Ratte. Bei diesem Modell lösen z. B. LNG und 3-Keto-Desogestrel aufgrund ihrer androgenen Potenz eine dosisabhängige Gewichtszunahme der ventralen Prostata aus [33]. Die relative Bindungsaffinität von LNG und von Desogestrel beträgt 70 bzw. $40 \%$ derjenigen von Testosteron. In einem ähnlichen Experiment wurde gezeigt, dass auch das Progesteronderivat MPA bei hoher Dosierung eine Androgenpotenz besitzt, während für Nomegestrol-Acetat wie auch für Progesteron selbst bei sehr hoher Dosierung keine androgene Wirkung nachgewiesen werden konnte [12]. Dies bedeutet, dass das Pregnan MPA im Gegensatz zum Norpregnan Nomegestrol-Acetat eine gewisse androgene Potenz besitzt, die aber niedriger ist als die von LNG und ande- 
Tabelle 2

Hormonale Gestagenpotenz und Inaktivierung von hepatischen mikrosomalen Cytochrome-P450-abhängigen Enzymen in vitro. (Nach [31])

\begin{tabular}{|c|c|c|c|}
\hline Gestagen & $\begin{array}{l}\text { TFD } \\
\text { [mg/Zyklus] }\end{array}$ & $\begin{array}{l}\text { OID } \\
\text { [mg/Tag] }\end{array}$ & $\begin{array}{l}\text { AA-Aktivität } \\
\text { [\%] }\end{array}$ \\
\hline Progesteron & 4200 & 300 & \\
\hline Medroxyprogesteron-Acetat & 50 & & \\
\hline Megestrol-Acetat & 50 & & \\
\hline Chlormadinon-Acetat & 25 & 1,7 & 30 \\
\hline Cyproteron-Acetat & 20 & 1,0 & 100 \\
\hline Dienogest & 6 & 1,0 & 40 \\
\hline Tibolon & & 2,5 & \\
\hline Norethisteron & 120 & 0,4 & \\
\hline Norethisteron-Acetat & 50 & 0,5 & \\
\hline Norgestimat & 7 & 0,2 & \\
\hline Levonorgestrel & 5 & 0,06 & \\
\hline Desogestrel/3-Keto-Desogestrel & 2 & 0,06 & \\
\hline Gestoden & 3 & 0,04 & \\
\hline Drospirenon & 50 & 2,0 & 30 \\
\hline Nomegestrol-Acetat & 100 & 5,0 & 90 \\
\hline Promegeston & 10 & 0,5 & \\
\hline
\end{tabular}

TFD Transformationsdosis bei der Frau, OID Ovulationshemmdosis bei der Frau (ohne Kombination mit einem Östrogen), AA-Aktivität relative antiandrogene Aktivität bei kastrierten, androgenbehandelten Ratten.

ren klassischen Testosteronderivaten. Auch das klinisch nur parenteral genutzte Norpregnan Nestoron zeigt keine signifikante Bindung an den Androgenrezeptor und hat somit keine androgene Partialwirkung [33].

Gestagene mit einer aktivierenden Bindung an den Androgenrezeptor und somit agonistischer androgener Wirkung führen nicht nur zu klinisch relevanten androgenen Nebenwirkungen wie Akne, fettiger Haut, Alopezie oder bei hoher Dosierung Hirsutismus, sondern sie können auch gewisse östrogenabhängige Stoffwechselvorgänge beeinflussen und so z. B. zu einer Veränderung des Lipidprofils mit HDL-Abfall und LDL-Anstieg führen. Gestagene mit androgener Partialwirkung beeinflussen auch bestimmte Hämostasefaktoren und die Synthese von in der Leber synthetisierten Proteinen wie Angiotensinogen. Auch bei der Synthese der Trägereiweiße SHBG, TBG oder CBG hemmen sie die stimulierende Wirkung der Östrogene.

\section{Antiandrogene Aktivität}

Die antiandrogene Aktivität wird durch mehrere Tests festgelegt. Zu den Gestage- nen mit einer antagonistischen Wirkung gehören die Antiandrogene Cyproteronacetat (CPA), Chlormadinonacetat (CMA), Dienogest und Drospirenon. Die relative antiandrogene Aktivität kann durch die Hemmung des Wachstums des Hahnenkamms oder bei kastrierten androgenbehandelten Ratten durch eine Abnahme des Prostatagewichtes ermittelt werden. Antiandrogene Gestagene üben ihre Wirkung via verschiedene Mechanismen aus: Einerseits können sie eine kompetitive Hemmung am Androgenrezeptor bewirken, andererseits binden sie an das Enzym 5- $\alpha$-Reduktase und behindern somit die Konversion von Testosteron in dessen aktiveren Metaboliten Dihydrotestosteron. Werden nichtandrogene Gestagene mit Östrogenen kombiniert, so neutralisieren sie den Östrogeneffekt auf die Trägereiweiße SHBG, CBG und TBG nicht. Die Folge des SHBG-Anstiegs ist eine vermehrte Bindung der zirkulierenden Androgene und somit weniger biologisch aktives freies Testosteron am Androgenrezeptor. Daraus resultiert ein therapeutisch günstiger Effekt von antiandrogenen Gestagenen bei Akne oder Hirsutismus.

\section{Antimineralokortikoide Partialwirkung}

Wie das natürliche Progesteron üben bestimmte Gestagene, insbesondere Drospirenon, eine antimineralokortikoide Partialwirkung aus, welche sich in einem kompensatorischen Anstieg der Aldosteronsekretion widerspiegelt. Eine aktivierende Bindung an den Mineralkortikoidrezeptor führt zu einer verminderten Wasserretention und damit zu einer Gewichtsreduktion. Eine solche antimineralokortikoide Wirkung ist v. a. Gestagenen eigen, welche dem natürlichen Progesteron gleichen.

\section{Glukokortikoide Partialwirkung}

Gestagene mit einer glukokortikoiden Partialwirkung können bei höherer Konzentration die ACTH-Sekretion hemmen. In klinisch gebräuchlichen Dosierungen besitzen sie eine gewisse glukokortikoide Wirkung auf die Gefäßwand (s. unten) und auf das Immunsystem. Eine solche glukokortikoidähnliche Partialwirkung findet sich sowohl bei 17-Hydroxyprogesteron- als auch bei Testosteronderivaten. Eine aktivierende Bindung an den Glukokortikoidrezeptor führt zu Salz- und Wasserretention mit Auswirkung auf Gewicht und Blutdruck und zu Blähungen.

\section{Aspekte bei der Gestagenauswahl}

Die in der Literatur zitierten Angaben zur Potenz eines Gestagens [31, 49, 50] beruhen allerdings meist nur auf relativ kleinen Untersuchungskollektiven. Es muss auch unterstrichen werden, dass die bei den üblichen In-vitro-Tests beobachteten Bindungsaffinitäten nicht immer gut mit den In-vivo-Assays zur östrogenen oder androgenen Partialwirkung korrelieren. Welches Gestagen soll nun wann eingesetzt werden? Wenn wir von den sehr unterschiedlichen Partialwirkungen der einzelnen Gestagene ausgehen, so folgt daraus, dass wir für den klinischen Einsatz ihre spezifischen Aktivitäten berücksichtigen müssen. Dabei müssen vor allem 3 Aspekte geklärt werden:

1. Hat das Gestagen eine progestative Wirkung und somit die Fähigkeit, das Endometrium in seine sekretorische Phase zu transformieren und eine Schwangerschaft zu erhalten? 


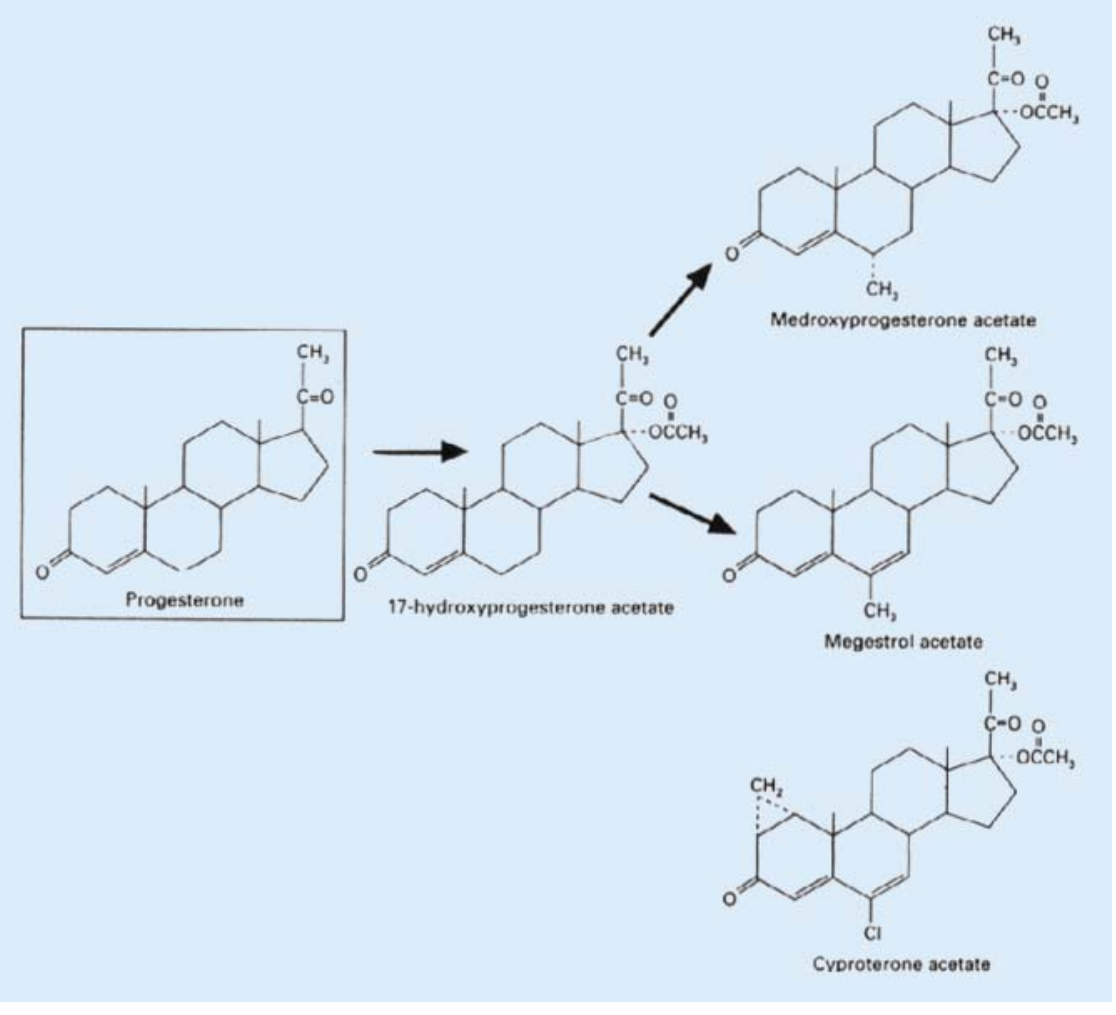

Abb. 3 \ 17-a-OH-Derivate

2. Hat das Gestagen eine antiöstrogene Wirkung und kann es somit die Östrogenrezeptoren herunterregulieren und die Höhe eines unter Östrogeneinfluss aufgebauten Endometriums reduzieren? Die antiöstrogene Wirkung von Gestagenen am Endometrium geht mit einer Suppression der Östrogenrezeptoren sowie der Aktivierung bestimmter Enzyme einher, welche - wie $17 \beta$-HSD Typus II Östradiol zu Östron metabolisieren, und der Östronsulfotransferase, welche Östron konjugiert.

3. Hat das Gestagen eine antiandrogene Wirkung, hemmt es also im Tierexperiment ein androgeninduziertes Prostatawachstum und wirkt es beim Menschen der endogenen androgenen Wirkung entgegen?

\section{Spezifische Aktivitäten der einzelnen Gestagene}

Die Partialwirkungen und die hormonale Potenz der im Folgenden beschriebenen Gestagene sind aus den Referenzen $[31,49,50]$ entnommen und in $\bullet$ Tabelle 1 und 2 zusammengefasst.

\section{7-a-OH-Progesteronderivate ohne antiandrogene Wirkung (Pregnane)}

Medroxyprogesteronacetat (MPA). MPA wird bei sequenzieller Gabe zur Hemmung der Endometriumproliferation üblicherweise in einer Dosierung von 5$10 \mathrm{mg} /$ Tag per os eingesetzt. Bei kontinuierlich kombinierter Gabe genügen 2,5 mg MPA pro Tag. MPA bindet sich nicht an die Trägereiweiße sexhormonbindendes Globulin (SHBG) und kortisonbindendes Globulin (CBG). Die Bindung an Albumin beträgt $88 \%$ [49]. MPA besitzt somit eine äußerst hohe Bioverfügbarkeit von beinahe $100 \%$. Obwohl MPA eine gewisse Bindungsaffinität zum Aldostonrezeptor besitzt, hat MPA in niedrigerer Dosierung klinisch keine mineralokortikoide oder antimineralokortikoide Aktivität. MPA antagonisiert den östrogeninduzierten Anstieg der Triglyzeride und des HDL-Cholesterins nicht, kann aber bei alleiniger Gabe (z. B. als Depot-MPA) HDL absenken [30]. MPA hat keine antiandrogene Wirkung. Es besitzt sogar eine geringe androgene Potenz, welche bei alleiniger MPA-Gabe zum Tragen kommen kann. Lange Zeit unterschätzt wurde die glukokortikoide Wirkung, welche MPA dank seiner Bindung an den Glukokortikoidrezeptor besitzt. Diese glukokortikoide Wirkung kann zu einer Aufregulierung des Thrombinrezeptors und einer Stimulierung der prokoagulatorischen Aktivität [24] und somit zu vaskulären Nebenwirkungen führen (s. unten). Bei höherer Dosierung kann MPA die Glukosetoleranz einschränken, jedoch ohne den Lipidstoffwechsel zu verändern [54].

Megestrolazetat. Megestrolazetat ist strukturell dem MPA äußerst ähnlich, hat wie MPA eine hohe Bioverfügbarkeit von beinahe $100 \%$ und keine Bindungsaffinität an SHBG oder CBG und somit auch ein ähnliches Partialwirkungsprofil.

Medrogeston. In Gegensatz zu MPA, CMA und CPA besitzt Medrogeston eine Methylgruppe an $\mathrm{C} 17$-aund ist somit kein „echtes“ 17- $\alpha$-OH-Progesteronderivat. Seine Bioverfügbarkeit beträgt ca. 100\%. Für Medrogeston liegen wenig sichere Angaben bezüglich seiner Bindungsaffinitäten an die verschiedenen Steroidrezeptoren vor, doch scheint Medrogeston keine androgene Potenz zu besitzen. Die heute bekannten Angaben zu den Partialwirkungen von Medrogeston sind in $\bullet$ Tabelle 1 und 2 zusammengefasst.

\section{7-a-OH-Progesteronderivate mit antiandrogener Wirkung (klassische Antiandrogene)}

Cyproteron-Acetat (CPA) und Chlormadinon-Acetat (CMA) besitzen beide eine antiandrogene Aktivität (• Abb. 3, 4), wobei diejenige von CMA jedoch nur 20-30\% der antiandrogenen Potenz von CPA entspricht. Die antiandrogene Wirkung von CPA und CMA kommt durch eine kompetitive Hemmung von Androgenen am Androgenrezeptor zustande. Sie ist somit dosisabhängig.

Cyproteron-Acetat wird an Albumin gebunden und besitzt keine Bindungsaffinität an SHBG oder CBG; 93\% des CPA sind gebunden, $7 \%$ frei verfügbar. CPA besitzt daher eine Bioverfügbarkeit von beinahe $100 \%$. CPA wird im Fettgewebe gespeichert und von dort nur langsam freigesetzt, sodass bei längerer Einnahme hoher CPA-Dosen eine Akkumulation mit Depotwirkung resultiert. CPA besitzt bestimmte glukokortikoide Eigenschaften, 


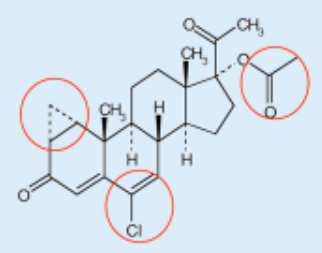

Cyproterone Acetate

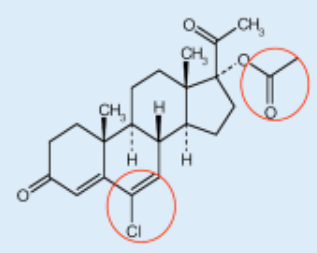

Chlormadinone Acetate

Abb.4 $\triangle$ Antiandrogen wirksame Gestagene

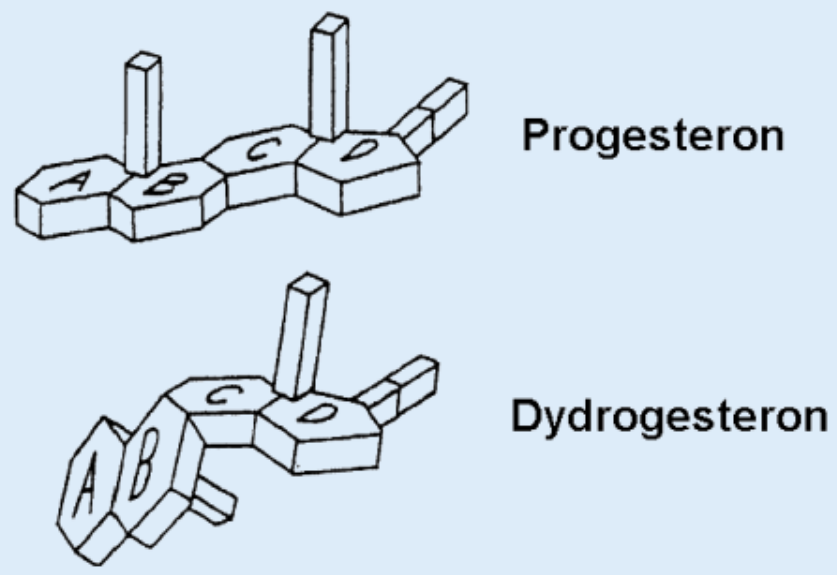

Abb. 5 Struktur der Retroprogesterone am Beispiel von Dydrogesteron

deren klinische Bedeutung allerdings noch unklar ist.

Auch Chlormadinon-Acetat bindet sich an Albumin (97-90\% zirkulieren gebunden) und besitzt keine Affinität zu SHBG oder CBG. Seine Bioverfügbarkeit ist daher ebenfalls hoch (praktisch 100\%). Da sein Metabolit 3- $\alpha$-hydroxy-CMA 70\% der antiandrogenen Aktivität von CMA besitzt, spielt der enterohepatische Kreislauf hier ein Rolle.

Die in den üblichen Kombinationspräparaten verwendete Dosierung von $1 \mathrm{mg}$ (substitutiv) bzw. $2 \mathrm{mg}$ (kontrazeptiv) für CPA und 2 mg für CMA (kontrazeptiv) garantieren eine ausreichende gestagene Wirkung auf das Endometrium.

\section{Retroprogesterone}

Retroprogesterone (• Abb. 5) haben typischerweise eine andere sterische Konfiguration ihres Moleküls als Progesteron (• Abb. 3). Während die Ringe A-D, welche das Progesteronmolekül bilden, in einer Ebene liegen, ist der B-Ring bei den Retroprogesteronen gegenüber dem C-Ring in einem $60^{\circ}$-Winkel abgeknickt. Dydroges-
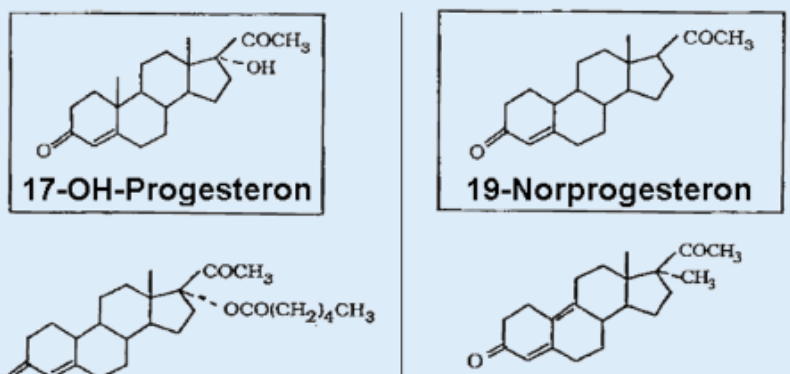

$$
\text { Gestonoron-Caproat }
$$<smiles>COC(=O)C1(C)CCC2C3C=C(C)C4=CC(=O)CCC4C3CCC21C</smiles>

Nomegestrol-Acetat

\section{Demegeston}<smiles>CCOC(=O)C1(C)CCC2C3CCC4=CC(=O)CCC4=C3CCC21C</smiles>

Promegeston

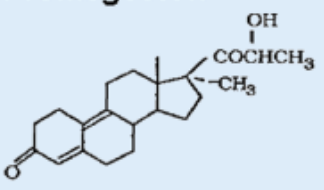

Trimegeston

Abb. $6 \Delta$ Struktur der Norpregnane und von Nomegestrol-Acetat teron ist heute das einzige klinisch verwendete Retroprogesteron. Es bindet wegen seiner Konfiguration praktisch ausschließlich an den Progesteronrezeptor. Retroprogesterone gelten deshalb als metabolisch gesehen „neutrale“ synthetische Gestagene: Werden 2,5, 5, 10 oder 20 mg Dydrogesteron fest kombiniert $1 \mathrm{mg}$ Östradiol beigegeben, so kommt es zu einem LDL-Abfall und einem HDL-Anstieg. Wegen der besseren Bioverfügbarkeit und der progestativen Aktivität seiner Metaboliten ist die Äquivalenzdosis zu Progesteron trotz einer schlechteren Bindungsaffinität an den Progesteronrezeptor signifikant niedriger und beträgt, auf das Endometrium bezogen, $10-20 \mathrm{mg} /$ Tag. Dydrogesteron führt in seiner üblichen Dosierung von (je nach Behandlungsschema) 5-20 mg pro Tag zu keinem Temperaturanstieg.

\section{Norpregnane}

Norpregnane (• Abb. 6) sind 19-Norprogesteronderivate ( $\bullet$ Abb. 7). Sie besitzen keine Methylgruppe an Position 19. Ihre hormonellen Charakteristika gleichen denjenigen der klassischen Progesteronde- rivate. Norpregnanderivate werden v. a. in Frankreich klinisch eingesetzt.

Promegeston. Promegeston ist ein potentes Gestagen und Antiöstrogen mit einer kurzen Halbwertszeit und besitzt weder eine androgene noch eine antiandrogene, aber eine schwache glukokortikoide Partialwirkung. Promegeston bindet nicht an SHBG und schwach an CBG, und wird v. a. von Albumin transportiert. Promegeston hat klinisch ein neutrales Wirkungsprofil. Bei der Beigabe von 0,5 mg Promegeston/Tag zu Östradiol (perkutan) verhalten sich die Serumlipide und -lipoproteine sowie die Serumspiegel von SHBG, Angiotensinogen, Antithrombin gleich wie unter der Kombination von Progesteron mit perkutanem Östradiol: Alle Werte bleiben unverändert [51].

Trimegeston. Trimegeston wurde von allen Norpregnanderivaten zuletzt in den klinischen Gebrauch übernommen. Es ist ein aktiver Metabolit von Promegeston. Von allen Norpregnanderivaten ist Trimegeston das Gestagen mit der höchsten Po- 
tenz. Es führt bereits in einer täglichen Dosis von o,1 mg bei einem östrogenstimulierten Endometrium zu einer sekretorischen Transformation. Bei zyklischer Kombination mit Östradiol wird Trimegeston in einer Dosierung von 0,25-0,5 mg pro Tag eingesetzt. Zykluskontrolle und Nebenwirkungen entsprechen ungefähr derjenigen von $2 \mathrm{mg}$ Östradiol kombiniert mit 0,5 mg Norgestrel oder $1 \mathrm{mg}$ NETA $[2,37]$, wobei einzig die Blutungsdauer etwas kürzer ist. Trimegeston besitzt keine glukokortikoide, keine androgene und keine antiandrogene Partialwirkung, weist hingegen eine schwache antimineralokortikoide Aktivität auf $[2,44,56]$. Trimegeston neutralisiert die östrogeninduzierten Veränderungen des Lipidstoffwechsels nicht.

Nestoron. Nestoron hat eine sehr niedrige orale Bioverfügbarkeit von nur $10 \%$, was eine Folge seiner kurzen Halbwertszeit von nur 1-3 h ist. Wegen der fehlenden Bindung an SHBG ist die Halbwertszeit kürzer als diejenige der meisten anderen Gestagene. Deshalb wird Nestoron praktisch ausschließlich parenteral in einer Slow-Release-Form als subdermales Implantat oder als transdermales Gel eingesetzt [39]. Nestoron bindet nicht an den Androgenrezeptor. Es besitzt keine glukokortikoide Partialwirkung, obwohl es an den Glukokortikoidrezeptor bindet. Im Ovulationshemmtest ist Nestoron 3-mal potenter als Levonorgestrel, wenn es parenteral verabreicht wird [33].

Nomegestrolacetat. Nomegestrolacetat unterscheidet sich von Megestrolazetat durch das Fehlen der Methylgruppe in Position 19. Es gilt daher chemisch gesehen streng genommen als 17- $\alpha$-Hydroxy-19Norprogesteron-Derivat. Es besitzt ein lange Halbwertszeit von 35-50 h. Nomegestrolacetat ist $\mathrm{zu}_{36 \%}$ an SHBG und $61 \%$ an $\mathrm{Al}$ bumin gebunden [37]. Seine Transformationsdosis am Endometrium beträgt $5 \mathrm{mg}$. Nomegestrolacetat besitzt eine ausgeprägte antiandrogene Wirkung, hat aber keine glukokortikoide, antimineralokortikoide oder androgene Aktivität. Seine antiandrogene Potenz liegt zwischen derjenigen von CMA und CPA. Eine Dosis von $5 \mathrm{mg}$ Nomegestrolacetat pro Tag induzierte keine Veränderungen der Serumspiegel von SHBG, CBG, Angiotensinogen, HDL-
Cholesterol, LDL-Cholesterol, Fibrininogen oder Plasminogen, führte aber zu einem Anstieg von Antithrombin und senkte die Triglyzeride $[6,43]$. Die Beigabe von Nomegestrolacetat zu einer Östrogenbehandlung neutralisierte die östrogeninduzierten Effekte auf den Lipidstoffwechsel nicht $[43,51]$.

\section{Nortestosteronderivate}

Norethisteron (NET) und Norethistoronacetat (NETA). In den meisten bei uns eingesetzten Präparaten findet sich Norethisteronacetat, das im Intestinaltrakt und in der Leber rasch zu Norethisteron hydrolysiert wird. Somit können Pharmakokinetik und -dynamik für NET und NETA gemeinsam betrachtet werden. Die orale Bioverfügbarkeit von NET und NETA ist $40-80 \%$, beeinflusst von der Partikelgröße und somit der Galenik des verabreichten Präparates. NET wird zu 36\% an SHBG und zu 61\% an Albumin gebunden [52]. Bei einer Dosis von 2 mg NET pro Tag kommt es zu einer gewissen Akkumulation der Substanz. Die Halbwertszeit beträgt $1,5 \mathrm{~h}$ ( $\mathrm{t}_{1 / 2}$ alpha $)$ bzw. 9,5 h ( $\left.\mathrm{t}_{1 / 2 \text { beta }}\right)$. Klinisch bedeutend ist die Tatsache, dass o,35\% von NET in der Leber zu EE aromatisiert werden [31]. Bei einer niedrigen Dosierung von $1 \mathrm{mg}$ NET kommt dieser Aromatisierung in Gegenwart von natürlichem 17- $\beta$-Östradiol keine klinische Relevanz zu. Werden aber NET-Dosen von 5 und $10 \mathrm{mg}$ per os verabreicht, so entstehen EE-Spiegel, die denjenigen einer Einnahme von 30 bzw. $60 \mu \mathrm{g}$ EE entsprechen [32].

Obwohl Norethisteron wie alle Nortestosteronderivate (• Abb. 7) vom anabolen Nandrolon (=19-Nortestosteron) abgeleitet ist, besitzt NET nur noch eine schwache androgene Partialwirkung, die aber bei entsprechender Veranlagung klinisch zu einer leichten Akne führen kann. NET hat keine glukokortikoide und keine antimineralokortikoide Aktivität. Oral eingenommen, hat NETA eine gewisse neutralisierende Wirkung auf den günstigen metabolischen Effekt von Östradiol. Bei transdermaler Verabreichung von Östradiol und NETA fehlt diese ungünstige Partialwirkung [6o]. Transdermales NETA führt im Gegensatz zu seiner peroralen Verabreichung zu keiner Beeinträchti- gung des Kohlenhydratstoffwechsels und einer nur geringen Abnahme des totalen Cholesterins, des LDL-Cholesterins, des HDL-Cholesterins und der Apolipoproteine B und A1. Dagegen kommt es zu einem starken Anfall der totalen Triglyzeride. In der üblichen transdermalen Dosierung werden vom verwendeten Patch o,25 mg NETA an die Haut abgegeben. Dadurch kann bei einer transdermalen Östrogendosis von $50 \mu \mathrm{g}$ mit sequenzieller NETA-Gabe eine Endometriumhyperplasie in $98 \%$ verhindert werden, das Blutungsmuster ist in ca. $80 \%$ regelmäßig. NETA kann die günstige Wirkung von Östrogenen auf den Knochen verstärken.

Levonorgestrel und Norgestrel. Norgestrel ist ein Racemat mit gleich viel inaktivem Dextronorgestrel und aktivem Levonorgestrel. Somit ist die hormonelle Aktivität von $0,5 \mathrm{mg}$ Norgestrel identisch mit derjenigen von 0,25 mg Levonorgestrel (LNG). Levonorgestrel ist $\mathrm{zu} 48 \%$ an SHBG und 50\% an Albumin gebunden. Die Halbwertszeit von LNG beträgt $1 \mathrm{~h}$ ( $\left.t_{1 / 2 \text { alpha }}\right)$ bzw. $24 \mathrm{~h}\left(\mathrm{t}_{1 / 2 \text { beta }}\right)$. LNG ist ein potentes Gestagen ohne glukokortikoide und ohne antimineralokortikoide Partialwirkung, besitzt aber wie NETA eine geringe androgene Aktivität. Wegen dieser androgenen Aktivität führt die alleinige orale Gabe von LNG zu einer Reduktion der SHBG-Spiegel, wogegen die Kombination mit einem Östrogen in einem SHBG-Anstieg resultiert.

LNG besitzt eine östrogene Partialwirkung, wie dies im Test der Anstieg des Uterusgewichts immaturer Ratten zeigt. Diese wird durch LNG, aber nicht durch $\mathrm{Ne}$ storon, bei vergleichbarer Dosierung signifikant erhöht [33], obwohl weder LNG noch Nestoron eine Bindung an den Östrogenrezeptor zeigen.

Wird eine Kombination von Östradiol und NLG transdermal verabreicht, so kommt es zu keiner Veränderung der Serumlipide und Lipoproteine.

Intrauterin wird LNG mit Hilfe eines IUD verabreicht. Die auf dem Markt befindliche hormonfreisetzende Spirale enthält 52 mg LNG, was eine Freisetzung von LNG über 5 Jahre garantiert. Im 1 . Jahr werden aus dem IUD $20 \mu \mathrm{g}$ LNG/Tag und im 5. Jahr noch $15 \mu \mathrm{g}$ LNG/Tag freigesetzt [31, 46]. Dieses gestagenhaltige IUD wird pri- 
mär zur Kontrazeption eingesetzt, kann jedoch auch als Gestagenkomponente bei einer hormonalen Substitution nach der Menopause verwendet werden. Wird ein LNG-IUD mit einer Freisetzung von $20 \mu \mathrm{g}$ NLG pro Tag zusammen mit $2 \mathrm{mg}$ Östradiolvalarat oral verwendet, so findet sich ein signifikanter Anstieg des HDLCholesterins und eine Abnahme des totalen Cholesterins, des LDL-Cholesterins und des Lipoprotein (a). Die günstige Wirkung auf HDL-Cholesterin wird mit einem 10- $\mu$ g-LNG-IUD über 12 Monate aufrechterhalten, dagegen mit dem $20 \mu \mathrm{g}$ NLG-IUD innerhalb weniger Monate neutralisiert [46].

Norgestimat. Norgestimat ist eine Prodrug. Es wird über 2 Stufen im Intestinaltrakt und der Leber via LNG-3-Oxym und LNG-17 $\beta$-Azetat in LNG abgebaut. Die hormonell aktiven Metaboliten von Norgestimat sind LNG und LNG-3-Oxym (Norelgestromin). Im Gegensatz zu LNG sind Norgestimat und Norelgestromin nicht an SHBG und CBG gebunden. Da LNG Endmetabolit und gleichzeitig aktiver Metabolit ist, entsprechen die Partialwirkungen von Norgestimat weitgehend denjenigen von $\mathrm{LNG}$.

Desogestrel. Desogestrel ist eine Prodrug, dessen maßgebender aktiver $\mathrm{Me}$ tabolit das 3-Keto-Desogestrel ist. Die Bioverfügbarkeit beträgt 76\%; 32\% zirkulieren an SHBG gebunden, $66 \%$ binden sich an Albumin. 3-Keto-Desogestrel (=Etonogestrel) besitzt eine antiöstrogene und eine androgene, jedoch keine glukokortikoide oder antimineralokortikoide Wirkung. Im Bioassay bei der immaturen Ratte beträgt die androgene Wirkung auf die Gewichtszunahme der ventralen Prostata noch 40\% derjenigen von Testosteron. Nach Nestoron besitzt 3-Keto-Desogestrel das beste Verhältnis zwischen den Bindungsaffinitäten für den Progesteron- und den Androgenrezeptor. Diese residuelle androgene Wirkung kann zu leichter Akne führen, wenn Desogestrel allein verabreicht wird. In Kombination mit einem Östrogen übt Desogestrel keine klinisch relevanten negativen Effekte auf das Lipidmuster oder den Kohlehydratstoffwechsel aus.
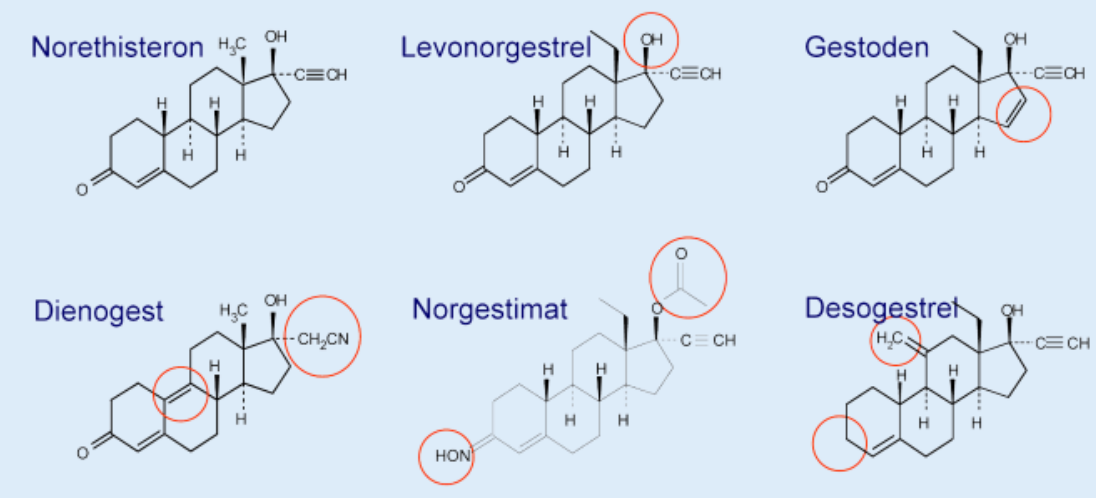

Abb.7 $\triangle$ 19-Nortestosteron-Derivate

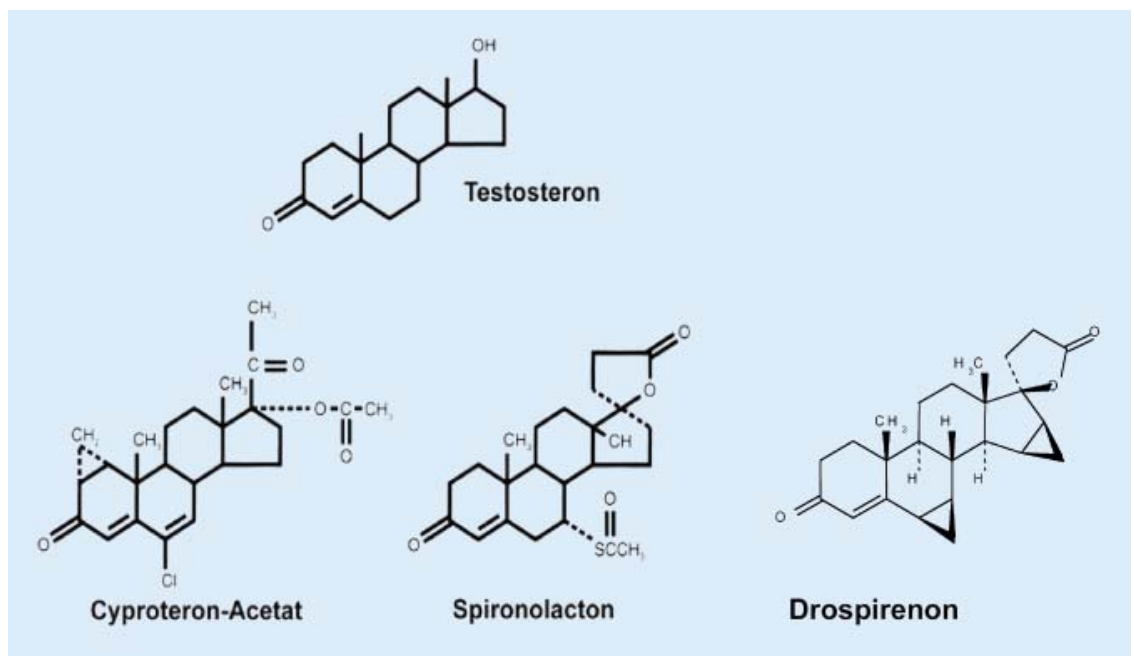

Abb. $8 \Delta$ Struktur von Drospirenon im Vergleich zu derjenigen von Testosteron, Cyproteron-Acetat und Spironolacton

Gestoden. Gestoden ist im Gegensatz zu Desogestrel und Norgestimat keine Prodrug. Seine Bioverfügbarkeit beträgt beinahe $100 \%$. Gestoden hat eine antiöstrogene, eine androgene, eine glukokortikoide und eine antimineralokortikoide Partialwirkung. Auch Gestoden gilt in Kombination mit einem Östrogen als weitgehend stoffwechselneutral.

Die Drittgenerationsgestagene Desogestrel und Gestoden wurden gegenüber den Gestagenen der 2. Generation mit einem leicht erhöhten thromboembolischen Risiko in Verbindung gebracht. Orale Kontrazeptiva mit einem weniger androgenen oder antiandrogenen Gestagen wie Gestoden, Desogestrel, CPA haben in bestimmten Studien gegenüber LNG ein leicht erhöhtes Risiko von thromboembolischen Ereignissen gezeigt. Ein denkbarer Mechanismus könnte sein, dass Gestagene mit stärkerer androgener Potenz eine gerin- gere Veränderung im Gleichgewicht der hepatischen Proteine des Gerinnungssystems auslösen [31]. Nach Lidegaard [35] beträgt das RR 1,3 und liegt mit seinem Vertrauensintervall an der Grenze der Signifikanz $(1,0-1,8 ; p<0,05)$. Da andere Daten das Vorliegen eines erhöhten relativen $\mathrm{Ri}$ sikos nicht bestätigen konnten und da die Unterschiede - sofern überhaupt vorhanden - klinisch bei gesunden jungen Frauen nicht relevant sind, besteht kein Grund, die $\mathrm{OH}$ mit den gut bekannten, meist gut ertragenen und zuverlässigen Drittgenerationsgestagenen zu meiden. Das am meisten verbreitete kontrazeptive reine Gestagenpräparat verwendet mit Desogestrel ein Gestagen der 3. Generation, ebenso das am meisten gebräuchliche kontrazeptive Implantat.

Dienogest. Dienogest ist ein antiandrogenes Gestagen mit starker gestagener Par- 
tialwirkung. Dienogest ist in einigen Ländern sowohl in oralen Kontrazeptiva als auch in Präparaten zur Hormonsubstitution eingesetzt, jedoch nicht als Reinsubstanz erhältlich. Dienogest besitzt anstelle der Ethinylgruppe an Position C17-a eine Cyanomethylgruppe und im B-Ring zwischen $\mathrm{C}_{9}$ und $\mathrm{C}_{10}$ eine Doppelbindung. Dienogest leitet sich von der Estrangruppe ab [42]. Da Dienogest keine androgene Partialwirkung besitzt wie die üblichen Testosteronderivate, wird es in seinem Wirkungsprofil als nahe der Pregnangruppe eingestuft. Dienogest ist daher das einzige Nortestosteronderivat, das nicht nur keine androgene, sondern im Gegensatz dazu eine antiandrogene Partialwirkung aufweist, die ungefähr 30\% derjenigen von CPA entspricht. Seine orale Bioverfügbarkeit liegt bei 90-95\%. Dienogest besitzt am Endometrium eine starke gestagene Wirkung, obwohl seine Bindungsaffinität zum Progesteronrezeptor relativ niedrig ist: Die Transformationsdosis von $6,3 \mathrm{mg}$ pro Zyklus ist derjenigen von LNG vergleichbar. Dies mag daher kommen, dass die freie Fraktion von Dienogest in der Zirkulation als Folge der fehlenden Bindung an SHBG oder CBG 10\% beträgt; 90\% sind an Albumin gebunden. Wird Dienogest mit Ethinylöstradiol kombiniert (2 mg Dienogest pro Tag plus $30 \mu \mathrm{g}$ EE pro Tag) resultiert daraus ein sicheres kombiniertes orales Kontrazeptivum mit einem Pearlindex von ungefähr 0,2 $[17,57]$. Dienogest besitzt eine östrogene, glukokortikoide und antimineralokortikoide Partialwirkung. Es antagonisiert die östrogeninduzierten Veränderungen bestimmter in der Leber synthetisierten Serumproteine nicht [29].

\section{Spirolactonderivate}

Drospirenon. Drospironon (• Abb. 8) gehört in die Familie der Spirolactone und besitzt im Gegensatz zum Spironolacton neben der antimineralokortikoiden und antiandrogenen Wirkung auch eine starke Gestagenpotenz. Drospirenon kann somit in Kombinationspräparaten als Gestagen eingesetzt werden und hat dabei wegen seiner antimineralokortikoiden und antiandrogenen Partialwirkung eine günstige Wirkung auf die Haut und auf das Körpergewicht. Die chemische Struktur von Drospirenon, einem Derivat des $17 \alpha-$ Spirolaktons, gleicht derjenigen des $\mathrm{Al}$ dosteronantagonisten Spironolacton, der aber im Gegensatz zu Drospirenon keine gestagene Wirkung besitzt [41]. Drospirenon hat eine Bioverfügbarkeit von 76-85\% und besitzt keine Bindungsaffinität an SHBG oder CBG. Es wird im Blut mehrheitlich an Albumin gebunden transportiert. Drospirenon besitzt eine mittelstarke Bindungsaffinität an Progesteronrezeptor und eine hohe Bindungsaffinität an den Androgenrezeptor und den Mineralokortikoidrezeptor. Die gestagene Wirkung von Drospirenon am Endometrium entspricht ungefähr $10 \%$ derjenigen von LNG. Die starke antimineralokortikoide Wirkung von Drospirenon führt zu einem Anstieg der Natriumausscheidung, was aber durch den Anstieg der Plasmareninaktivität um 100\% und der Aldosteronspiegel um 65\% kompensiert wird [40]. Die androgene Aktivität von Drospirenon beträgt ungefähr 30\% derjenigen von CPA. Drospirenon besitzt keine östrogene und keine nachweisbare glukokortikoide Aktivität [8]. Wegen der fehlenden androgenen Partialwirkung neutralisiert Drospirenon den günstigen Östrogeneffekt auf den Lipidstoffwechsel nicht.

Das auf dem Markt befindliche Kombinationspräparat zur oralen Kontrazeption besteht aus $30 \mu \mathrm{g}$ Ethinylöstradiol und $3 \mathrm{mg}$ Drospirenon. Drospirenon ist als Gestagen auch in einem Präparat zu einer HRT enthalten (Drospirenon plus Östradiolvalerat). Drospirenon besitzt neben seiner Gestagenwirkung auch eine antimineralokortikoide und eine antiandrogene Partialwirkung. Es ist in der Lage, die stimulierende Wirkung von Ethinylöstradiol auf die Synthese von Angiotensinogen in der Leber weitgehend zu neutralisieren und damit den konsekutiven Anstieg der Natriumretention zu verhindern, sodass der unter einer Pille häufige leichte $\mathrm{Ge}$ wichtsanstieg in der Regel ausbleibt [14, $41,45]$. So wurde in einer 6-Monats-Studie mit Einnahme von einem Drospirenonund einem Levonorgestrelpräparat in der Drospirenongruppe (3 mg Drospirenon plus 20, 15 oder $30 \mu \mathrm{g}$ Drospirenon) eine Gewichtsreduktion von $0,7-1,7 \mathrm{~kg}$ und in der Levonorgestrelgruppe (150 $\mu \mathrm{g}$ Levonorgestrel plus $30 \mu \mathrm{g}$ EE) eine Gewichtszunahme von $0,7 \mathrm{~kg}$ Körpergewicht gefun- den. Dazu fand sich in der Drospironongruppe eine leichte Reduktion des Blutdrucks. Ovulationshemmung, Zykluskontrolle, Nebenwirkungsprofil und Sicherheit $[16,25,43,55]$ entsprechen den Erfahrungen mit den bekannten Mikropillen.

\section{Folgen der Gestagenpartialwirkungen}

Typisch für die Gestagene ist es, dass kleinste strukturelle Veränderungen sehr weit gehende Wirkungsverschiebungen mit sich bringen. So kann an der Grundstruktur des Hydroxyprogesterons der Wegfall des $\mathrm{CH}_{3}$-Radikals in Position 19 und eine zusätzliche Doppelbindung zwischen $\mathrm{C}_{6}-\mathrm{C}_{7}$ dem daraus resultierenden Nomegestrol-Acetat eine stärkere gestagene Potenz als dem älteren Medroxyprogesteron-Acetat verleihen, obwohl beides 17-a-Hydroxyprogesteron-Derivate sind [55]. Andere Veränderungen des Steroidgerüsts beeinflussen die orale Bioverfügbarkeit und somit die klinische Aktivität eine Moleküls: Nestoron, ein 19-Norprogesteron ohne $\mathrm{CH}_{3}$-Radikal in Position 6, ist zwar deutlich potenter als NomegestrolAcetat, kann aber nicht oral eingesetzt werden, da es von der Leber relativ rasch abgebaut wird [33].

Alle klinisch eingesetzten Gestagene besitzen definitionsgemäß eine Progesteronwirkung und sind somit wirksame $\mathrm{Ge}$ genspieler der proliferativen Östrogenwirkung auf das Endometrium.

\section{- Allerdings sind die gestagene Partialwirkung und die individuelle Bioverfügbarkeit der einzelnen Sub- stanzen stark unterschiedlich, sodass die Dosierungen, welche dazu not- wendig sind, um den erwünschten endometrialen Effekt zu erzielen, von wenigen Mikrogrammen zu mehreren Milligrammen reichen.}

Eine Folge dieser Unterschiede ist es, dass die „potenteren Moleküle“ in sehr niedriger Dosierung in lang wirksamen Abgabesystemen eingesetzt werden können. $\mathrm{Zu}$ diesen Systemen gehören Gels, Pflaster und IUDs.

Entsprechend ihrer Struktur und ihrem Ursprungsmolekül besitzen die einzelnen Moleküle neben der gestagenen 
verschiedene zusätzliche Partialwirkungen, von denen einige als günstig und andere als ungünstig und somit als mögliche Ursache von Nebenwirkungen eingestuft werden. Bereits die Auflistung dieser möglichen Unterschiede zeigt, dass es falsch ist, die verschiedenen Potenzen und $\mathrm{Ne}$ benwirkungen von Gestagenen als „Klasseneffekt" zusammenzufassen.

Die 17-Hydroxy-Progesteronderivate weisen unter sich sehr unterschiedliche Partialwirkungen auf. MPA besitzt eine geringe androgene Potenz. Bei höherer Dosierung weist MPA eine glukokortikoide Aktivität auf [22]. Megestrol-Acetat hat ebenfalls eine gewisse glukokortikoide Aktivität, die jedoch um rund die Hälfte geringer ist als diejenige von MPA. Demgegenüber ist Cyproteron-Acetat (CPA) ein stark antiandrogenes Gestagen mit einer klinisch vernachlässigbaren glukokortikoiden Partialwirkung.

Die 19-Norprogesterone gelten als besonders progesteronähnlich und besitzen bei den üblichen therapeutischen Dosierungen keinerlei androgene, östrogene oder glukokortikoide Partialwirkung $[12,33]$. In den letzten Jahren wurden aus dieser Gruppe mehrere neuere Gestagene entwickelt und eingeführt [48]. Diese sog. Norpregnane werden auch als "reine“ gestagene Moleküle angesehen, da sie weitgehend selektiv an den Progesteronrezeptor und wenig an die anderen Steroidrezeptoren binden [48]. Zu den Norpregnanen gehören das Promegestrol-, das Demegestrol-, das Trimegestrol- und das Nestorol. Wegen seines klinischen Profils wird das von der Wirkung und Struktur her verwandte Molekül Nomegestrol-Acetat meist dazugerechnet.

Nestoron ist wegen seiner schlechten peroralen Bioverfügbarkeit und wegen seines Verhaltens am Glukokortikoidrezeptor ein Sonderfall. Nestoron bindet zwar an den Glukokortikoidrezeptor, besitzt aber bei den durchgeführten In-vivo-Tests keine glukokortikoide Aktivität: Unter Nestoron kommt es zu keiner Zunahme des Leberglykogens und der Thyrosin-Transaminase TAT, welche beide unter Dexamethason signifikant ansteigen [33]. Andererseits besitzt Nesteron in hoher Dosierung bei der ovariektomierten weiblichen Ratte wie Glukokortikoide eine signifikante Wirkung auf die Thymusregression [33].
Alle 19-Nortestosteronderivate besitzen eine gewisse androgene Partialwirkung, wenn diese auch bei den Gestagenen der 3. Generation nur noch sehr schwach ausgeprägt ist, während nur wenige dieser Gestagene auch eine östrogene Partialwirkung besitzen. Die Partialwirkungen dieser Gruppe sind also relativ homogen.

Dienogest, eines der neueren Gestagene, ist das einzige Nortestosteronderivat, das keine androgene, sondern sogar eine antiandrogene Partialwirkung aufweist: obwohl sich Dienogest von den Estranen ableitet, besitzt es keine androgene Partialwirkung wie die klassischen Testosteronderivate. Es wird von seinem klinischen Wirkungsprofil nahe der Pregnangruppe eingereiht.

Auch Drospirenon gehört zu den neueren Gestagenen. Drospirenon, ein Spirolactonabkömmling, ist ein antimineralokortikoides Gestagen mit einer mittleren antiandrogenen Potenz [41]. Drospirenon besitzt ähnliche pharmakodynamische Eigenschaften wie das natürliche Progesteron $[18,45]$ und wurde zunächst als Gestagen zur oralen Kontrazeption entwickelt.

Obwohl alle Gestagene in genügend hoher Dosierung zu einer Ovulationshemmung führen, werden die potenteren Moleküle, bei denen bereits die Verabreichung einer kleinen Dosierung für die Ovulationshemmung ausreicht, speziell in lang wirksamen Abgabesystemen, wie z. B. Vaginalringen, Implantaten, transdermalen Pflastern oder Gels, eingesetzt.

\section{Problemfall: gestagene Partialwirkungen und kardiovaskuläre Erkrankungen}

Es ist aus heutiger Sicht denkbar, dass Gestagenpartialwirkungen neben zahlreichen anderen Faktoren über das vaskuläre Risiko mitentscheiden. Die meisten zur Kontrazeption eingesetzten Gestagene sind von Testosteron abgeleitet, sodass zahlreiche ihrer Nebeneffekte auf ihrer androgenen und z. T. auch ihrer glukokortikoiden Partialwirkung beruhen. Testosteronderivate werden in Europa v. a. in Skandinavien und auf dem europäischen Kontinent zur Hormonsubstitution nach der Menopause verwendet. Einige europäische Länder, wie z. B. Frankreich, ziehen allerdings andere Gestagene wie die
Norpregnane und das natürliche Progesteron vor. Wegen der unerwünschten androgenen Partialwirkung der klassischen Testosteronderivate ging der Trend in der Gestagenforschung seit Jahren dahin, diese Androgenpotenz zu senken. Bei den Testosteronderivaten ist dies mit der Entwicklung der 3. Generation weitgehend gelungen. Diese Gestagene besitzen eine deutlich schwächere androgene Wirkung als die Substanzen der 2. Generation. Der Preis ist aber bei kombinierten Ovulationshemmern eine geringere Neutralisierung der metabolischen Wirkung von EE. Die Frage ist noch offen, ob das durch die Senkung der androgenen Partialwirkung denkbare geringere arterielle Risiko durch ein leicht höheres thromboembolisches Risiko als Folge der unterschiedlichen Beeinflussung der hepatischen Gerinnungsfaktoren bezahlt werden müsste: Wegen der verminderten Androgenpotenz kommt es zu einer reduzierten Neutralisierung des thrombogenen Effekts von EE.

\section{(2) Thromboembolische Ereig- nisse treten v. a. im 1./2. Jahr der Verabreichung auf}

Die bei mehreren Testosteronderivaten vorhandene glukokortikoide Partialwirkung, die sich auch bei gewissen $17-\mathrm{a}-\mathrm{Hy}$ droxyprogestreron-Derivaten (v. a. dem MPA) findet, könnte andererseits das vaskuläre Risiko auf der arteriellen Seite steigern (s. unten). Auffallend bei der Verabreichung von oralen Kontrazeptiva ist die Beobachtung, dass thromboembolische Ereignisse v. a. im 1./2. Jahr der Verabreichung auftreten [28]. Zu Beginn der Anwendung scheint nach einigen Arbeiten ein leicht erhöhtes Risiko für Drittgenerationspräparate gegenüber Zweitgenerationspräparaten zu bestehen (postuliertes erhöhtes relatives Risiko zwischen Dritt- und Zweitgenerationspräparaten), wobei diese Sicht nicht unumstritten ist. Der in einigen Arbeiten beobachtete geringe Risikoanstieg könnte auch die Folge einer selektiven Verschreibung von Drittgenerationspräparaten sein. Andere Studien [15] finden zwischen den verschiedenen Gestagentypen keinen statistischen Unterschied in der Thromboseinzidenz, sodass diese Frage noch offen bleiben muss. 
Aufgrund von Daten aus Untersuchungen bei der Frau [19, 20, 21, 27, 34, 36, 53] und beim nicht-humanen Primaten $[1,4$, 10, 38, 58, 59] liegt eine starke Evidenz dafür vor, dass ein Östrogenmangel die Progredienz der Atherosklerose fördert [10, 20, 38]. Es wird aufgrund der gleichen Daten postuliert, dass eine zeitgerechte noch während der Perimenopause oder möglichst früh nach der Menopause - begonnene Östrogengabe (ERT) das Fortschreiten der Atherosklerose verhindert. Andererseits wurde mehrfach gezeigt, dass bei älteren Frauen ab 60-65 Jahren mit bereits vorbestehender Koronarsklerose eine kombinierte Hormontherapie (HT) keinen Nutzen mehr bringt $[26,47]$.

\section{Studienergebnisse}

Welche experimentelle Daten liegen heute vor? Östrogen- und Progesteronrezeptoren sind innerhalb der Gefäßwände und auf den diese auskleidenden Endothelzellen nachgewiesen worden. Aus verschiedenen In-vitro-Studien ist bekannt, dass Östradiol direkt an der Gefäßwand durch die Freisetzung von NO eine günstige Wirkung auslöst, indem es zu einer Erschlaffung der glatten Muskelzellen und damit einer Vasodilatation führt. Wird im Affenmodell zusätzlich zu einem Östrogen natürliches Progesteron oder Norethisteron-Acetat verabreicht, so wird dadurch die günstige Östrogenwirkung auf die Koronardilatation nicht blockiert $[10,38]$. Weitere Studien bestätigen beim Rhesusaffen, dass Östradiol allein oder in Kombination mit natürlichem Progesteron einen antiatherogenen Effekt besitzt, der nicht von den vorhandenen HDL-Cholesterinwerten abhängt. Somit scheint es wahrscheinlich, dass natürliches Progesteron auch beim Menschen den günstigen Östrogeneffekt nicht neutralisiert und die östradiolinduzierte Hemmung einer Progression der Arteriosklerose nicht beeinträchtigt [1].

Demgegenüber findet sich im Rhesusaffenmodell bei der Kombination Östradiol/ MPA eine Reduktion der günstigen gefäßdilatierenden Östrogenwirkung um 50\% $[10,38,59]$. Eine spätere Studie mit einer Kombination von konjugierten equinen Östrogenen (=CEE) und MPA, die beim Menschen einer Dosis von o,625 mg CEE pro Tag und 2,5 mg MPA pro Tag entsprä- che, brachte allerdings ein günstigeres $\mathrm{Re}$ sultat, indem die Gruppe mit kombinierter Gabe von CEE/MPA keine Neutralisierung des östrogeninduzierten Schutzes vor dem Fortschreiten der Atherosklerose gezeigt hatte: Das Resultat fiel unter CEE/ MPA gleich aus wie unter CEE allein. Allerdings war in dieser 2. Studie die MPADosis auf 2 Verabreichungen pro Tag verteilt worden [38].

Bei der Frau wird zur hormonalen Substitution nach der Menopause in den Vereinigten Staaten überwiegend die Kombination CEE/MPA eingesetzt. Alle großen RCT $[26,47]$ wurden mit dieser Kombination durchgeführt. Bei der Women's Health Initiative (WHI; [4, 47]) fällt auf, dass im Studienarm mit einer Monotherapie von CEE [4] weder ein Anstieg des Brustkrebsrisikos noch ein Anstieg des kardiovaskulären Risikos gefunden wurde, während bei den älteren Studienteilnehmerinnen im kombinierten Arm (CEE plus MPA) ein Anstieg beider Risiken eintrat [47]. Die Beobachtungen unter Östrogenmonotherapie stimmen ebenso wie die Teilresultate im kombinierten CEE/ MPA-Arm bei den jüngeren Studienteilnehmerinnen (<10 Jahre nach der Menopause) mit den oben diskutierten Daten beim Rhesusaffen [38] einerseits und den großen Beobachtungsstudien beim Menschen andererseits, wie z. B. der Nurses Health Study [20], gut überein, welche bei den zum Beginn der Hormongabe im Vergleich zur WHI rund 10 Jahre jüngeren Frauen kein erhöhtes kardiovaskuläres Risiko fanden, unabhängig davon, ob CEE allein [4] oder kombiniert mit MPA [47] verabreicht wurde. Neben dem verschiedenen Alter der Studienteilnehmerinnen unterscheiden sich die Nurses' Health Study und die Women's Health Initiative allerdings auch dadurch, dass in der Nurses Health Study die Gestagengabe mehrheitlich sequenziell erfolgte, während es sich in der WHI um ein kontinuierlich kombiniertes Behandlungsschema handelte.

\section{( Es gibt keinen Klasseneffekt}

Alle diese Daten weisen darauf hin, dass Östrogene unabhängig von einer Gestagengabe kardioprotektiv wirken können, wenn sie unmittelbar nach der Menopause verabreicht werden. Andererseits stim- men in der WHI die Daten des kombinierten CEE/MPA-Arms, welche bei älteren Frauen (Mittel 63 Jahre) keinen kardioprotektiven Effekt nachweisen konnten, wiederum mit den Beobachtungen beim Rhesusaffen überein: Wurde im Affenmodell mit der Hormongabe zu spät begonnen, fand sich kein Benefit mehr. Da in der WHI im Östrogen-allein-Arm [4] auch ältere Frauen keinen Risikoanstieg zeigten, bleibt jedoch ein Zweifel an der neutralen Rolle der Gestagene: Es stellt sich die Frage, ob die widersprüchlichen Resultate unter alleiniger und mit MPA kombinierter Östrogengabe evtl. doch durch die Beigabe des Gestagens und nicht durch einen Unterschied bei den Probandinnen bedingt sein könnte. Diskutiert wird eine thrombogene Partialwirkung bei Gestagenen mit Bindungsaffinität an den Glukokortikoidrezeptor, wie dies MPA besitzt [31], indem möglicherweise durch diese Partialwirkung der Thrombinrezeptor hinaufreguliert wird. Dies führt über verschiedene lokale Mechanismen zu einer Förderung der Atherosklerose, zu einer Stimulierung des extrinsischen Gerinnungssystems und schließlich zu einem erhöhten koronaren Thrombose- und Infarktrisiko.

MPA und moderne Gestagene wie 3Keto-Desogestrel oder Gestoden (aber auch das natürliche Progesteron) könnten nach In-vitro-Versuchen mit Gefäßwandzellen eine solche Aufregulierung des Thrombinrezeptors begünstigen, während Gestagene ohne Bindungsaffinität an den Glukokortikoidrezeptor, wie z. B. NETA, LNG und Dienogest, dies nicht tun. Der Einfluss von NETA auf das Fortschreiten der Arteriosklerose wurde beim Kaninchen untersucht $[3,9]$. Beide Arbeiten zeigen, dass eine Östrogengabe das Fortschreiten der Arteriosklerose verlangsamt, unabhängig davon, ob das Gestagen Norethisteron-Acetat dem Östradiol beigegeben wird. Dexamethason hat bereits in sehr niedriger Dosierung in vitro eine ähnliche Wirkung auf die Gefäßwand wie Gestagene mit Bindungsaffinität an die Glukokortikoidrezeptoren.

Obwohl zurzeit keine klinischen Daten vorliegen, um die Glukokortikoidhypothese zu stützen, weisen In-vitro-Daten darauf hin, dass es eher eine glukokortikoide als eine androgene Partialwirkung des Gestagens ist, die zur Neutralisierung des 
günstigen Östrogeneffektes auf die Gefäßwand führen könnte. Sollte sich dies bestätigen, so müsste Gestagenen ohne glukokortikoide Partialwirkung der Vorzug gegeben werden.

$\mathrm{Ob}$ der Unterschied zwischen dem kombinierten Studienarm (CEE plus MPA) und dem alleinigen CEE-Arm auf einer spezifischen athrogenen Nebenwirkung des MPA oder auf ganz anderen, vom Gestagen unabhängigen Faktoren beruht, bleibt zurzeit somit noch offen. Da sich die beiden Studienarme der WHI auBer durch die Beigabe des Gestagens in einem Studienarm auch in zahlreichen anderen Punkten unterscheiden, müssen weitere Daten abgewartet werden, um die Gestagenfrage sicher beantworten zu können.

Dass die FDA aufgrund der WHI, die mit einem einzigen Gestagen und einer einzigen Verabreichungsform (peroral) durchgeführt worden war, für alle Gestagene einen Klasseneffekt ableitete, ist für uns Europäer nicht nachvollziehbar. Die einzelnen Gestagene unterscheiden sich sehr deutlich in ihren Partialwirkungen, wie schon allein die obige Diskussion zur Bedeutung der glukokortikoiden Partialwirkung zeigt, und besitzen somit individuelle Wirkungsund Nebenwirkungsprofile.

\section{Korrespondierender Autor Prof. Dr. M. Birkhäuser}

Abteilung für Gynäkologische Endokrinologie und Reproduktionsmedizin,

Universitäts-Frauenklinik, Inselspital,

Effingerstrasse 102, 3010 Bern, Schweiz

E-Mail: Martin.Birkhaeuser@insel.ch

Interessenkonflikt: Keine Angaben

\section{Literatur}

3. Alexandersen $\mathrm{P}$, Haarbo $\mathrm{JH}$, Zandberg $\mathrm{P}$ et al. (2003) Lack of difference among progestins on the antiatherogenic effect of ethinyl estradiol: a rabbit study. Hum Reprod 18:1395-1403

4. Anderson GL, Hutchinson F, Limacher M et al. (2004) Effects of conjugated equine estrogen in postmenopausal women with hysterectomy. The Women's Health Initiative Randomized Controlled Trial. JAMA 291:1701-1712

6. Basdevant A, Pelissier C, Conard J et al. (1991) Effects of nomegestrol acetate $(5 \mathrm{mg} / \mathrm{d})$ on hormonal, metabolic and hemostatic parameters in premenopausal women. Contraception 44:599-605

10. Clarkson TB, Appt SE (2003) MPA and postmenopausal coronary artery atherosclerosis revisited. Steroids 68:941-951
12. Duc I, Botella J, Bonnet P et al. (1995) Anti-androgenic properties of nomegestrol acetate. Arzneim Forsch Drug Res 45:70-74

15. Farmer RDT, Lawrenson RA, Thompson CR et al. (1997) Population-based study of risk of venous thromboembolism associated with various oral contraceptives. Lancet 349:83-88

16. Foidart JM, Wuttke W, Bouw GM et al. (2000) A comparative investigation of contraceptive reliability. Eur J Contracept Reprod Health Care 5:124-134

17. Foster RH (1998) Dienogest. Drugs 56:825-833

20. Grodstein F, Stampfer MS, Colditz GA et al. (1997) Postmenopausal hormone therapy and mortality. N Engl J Med 336:1769-1775

21. Grodstein F, Manson JE, Colditz GA et al. (2000) A prospective, observational study of postmenopausal hormone therapy and primary prevention of cardiovascular disease. Ann Intern Med 133:933941

28. Kemmeren JM, Algra A, Meijers JCM et al. (2002) Effects of second and third generation oral contraceptives and their respective progestagens on the coagulation system in the absence or presence of factor $V$ leaden mutation. Thromb Haemost 65:187-196

29. Kuhl H (1996) Comparative pharmacology of newer progestogens. Drugs 51:188-215

30. Kuhl H (2001) Pharmacology of progestogens. Basic aspects - progesterone derivatives. Menopause Rev 6:9-1619

31. Kuhl H (2005) Pharmacology of estrogens and progestogens: influence of different routes of administration. Climacteric 8 [Suppl 1]:3-63

32. Kuhnz W, Heuner A, Hümpel M et al. (1997) In vino conversion of norethisterone and norethisterone acetate to ethinyl estradiol in postmenopausal women. Contraception 56:379-385

33. Kumar N, Koide SS, Tsong YY, Sundaram K (2003) Nestorone : a progestin with a unique pharmacological profile. Steroids 65:629-636

35. Lidegaard $\emptyset$, Edström B, Kreiner S (2002) Oral contraceptives and venous thromboembolism: a fiveyear national case-control study. Contraception 65:187-196

36. Manson JE, Hsia J, Johnson KC et al. (2003) Estrogen plus progestin and the risk of coronary heart disease. N Engl J Med 349:523-534

38. Mikkola TS, Clarkson TB (2002) Estrogen replacement therapy, atherosclerosis, and vascular function. Cardiovasc Res 15:605-619

40. Oelkers W, Berger V, Bolik A et al. (1991) Dihydrospirorenone, a new progestogen with antimineralocorticoid activity : effects on ovulation, electrolyte excretion, and the reninaldosterone system in normal women. J Clin Endocrinol Metab 73:837-842

41. Oelkers W, Foidart JM, Dombovicz N et al. (1995) Effects of the new oral contraceptive containing an antimineralocorticoid progestogen, drospirenone, on the reninaldosterone system, body weight, blood pressure, glucose tolerance and lipid metabolism. J Clin Endocrinol Metab 80:1816-1821

44. Philibert D, Bouchoux F, Degryse M et al. (1999) The pharmacological profile of a novel norpregnana progestin (trimegestone). Gynecol Endocrinol 13:316-326

46. Raudaskoski T, Tapanainen J, Tomas E et al. (2002) Intrauterine $10 \mu \mathrm{g}$ and $20 \mu \mathrm{g}$ levonorgestrel systems in postmenopausal women receicing oral oestrogen replacement therapy: xlinical, endometrial and metabolic response. Br J Obstet Gynaecol 109:136-144
47. Rossouw JE, Anderson GL, Prentice RL et al. (2002) Risks and benefits of estrogen plus progestin in healthy postmenopausal women: principal results from the Women's Health Initiative randomized controlled trial. J Am Med Assoc 288:321-333

48. Rübig A (2003) Drospirenon: a new cardiovascularactive progestin with antialdosterone and antiandrogenic properties. Climacteric 6 [Suppl 3]:49-54

49. Schindler AE, Campagnoli A, Druckmann R et al. (2003) Classification and pharmacology of progestins. Maturitas 46 [Suppl 1]:S7-S16

50. Sitruk-Ware R (2002) Progestogens in hormonal replacement therapy: new molecules, risks, and benefits. Menopause 9:6-15

57. Wiegratz I, Lee JH, Kutschera E et al. (2002) Effect of dienogest-containing oral contraceptives on lipid metabolism. Contraception 65:223-229

60. Wiseman LR, McTavish D (1994) Transdermal estradiol/norethisterone. A rewiew of its pharmacological properties and clinical use in postmenopausal women. Drugs Aging 4:238-256
Das komplette Literaturverzeichnis ...

... finden Sie in der elektronischen Version dieses Beitrags unter GynaekologischeEndokrinologie.de 